\title{
Ativação de plaquetas de eqüinos com laminite induzida e tratados com ketoprofeno, fenilbutazona e flunixin meglumina
}

\author{
[Platelets activation in equine with induced laminitis and treated with ketoprophen, \\ phenylbutazone and flunixin meglumin]

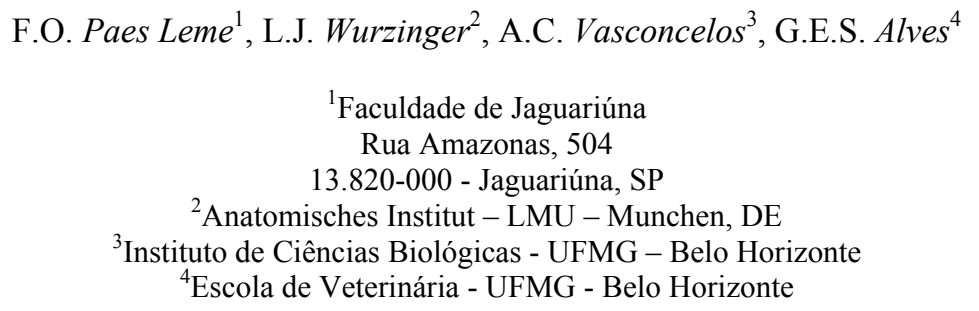

\section{RESUMO}

Avaliou-se a ativação de plaquetas em 20 eqüinos com laminite induzida, tratados com ketoprofeno, fenilbutazona e flunixin meglumina. As alterações de plaquetas incluíram mudança de forma, alteração da relação entre os eixos maior e menor, aumento de perímetro, emissão de pseudópodes, aumento no número de $\alpha$-grânulos e de grânulos de glicogênio e redução no número de $\gamma$-grânulos. As plaquetas de eqüinos, quando ativadas, apresentaram perfil de organela diferente de plaquetas normais, e as drogas antiinflamatórias, não-esteroidais, demonstraram atividade na ativação plaquetária de eqüinos in vivo. $\mathrm{O}$ flunixin meglumina apresentou melhor atividade em modular a ativação plaquetária de eqüinos in vivo do que a fenilbutazona e o ketoprofeno.

Palavras-chave: eqüino, laminite, plaquetas, ketoprofeno, fenilbutazona, flunixin meglumina

\begin{abstract}
The platelets activation from 20 equines submitted to laminitis induction and treated with ketoprophen, phenylbutazone and flunixin meglumin, was evaluated. The platelets changes included shape change, altered relations between axis, increased perimeter, pseudopodia, increased alpha-granules and glycogen-granules, and decreased in gamma-granules. Platelets when activated present a different organelle profile than normal ones. Equine activated platelets had different organelles profile than normal ones, and anti-inflammatory drugs can modulate the platelet activation, being the flunixin meglumin better than phenylbutazone and ketoprophen.
\end{abstract}

Keywords: equine, laminitis, platelets, ketoprophen, phenylbutazone, flunixin meglumin

\section{INTRODUÇÃO}

Apesar de seu tamanho reduzido, quando comparadas às outras células do sangue, as plaquetas são extremamente sensíveis a qualquer alteração física ou química do sangue ou do

Recebido em 24 de março de 2004 Aceito em 15 de setembro de 2005 E-mail: fabivet@yahoo.com Apoio: FAPEMIG sistema cardiovascular, respondendo prontamente a uma grande variedade de estímulos (Wurzinger, 1990; Klinger, 1997). Essa resposta rápida visa minimizar ou evitar as possíveis perdas de sangue, embora também possa estar associada ao reconhecimento e ataque a partículas estranhas e à modulação de respostas inflamatórias (Klinger, 1997) e alérgicas (Ablett et al., 1997). 
A ativação ocorre em poucos segundos e é acompanhada pela secreção do conteúdo dos grânulos das plaquetas (Klinger, 1997). A partir da ativação, ocorrem modificações físicas, químicas e estruturais - "metamorfose viscosa"que passam a sintetizar e secretar substâncias que potencializam a agregação celular hetero (Rinder et al., 1991b) e homotípica (Nyarko et al., 1998). A agregação é mediada pelo fibrinogênio plasmático, ou oriundo dos $\alpha$-grânulos de plaquetas ativadas, que se liga à glicoproteína IIb/IIIa das plaquetas (Meyers et al., 1979; Nyarko et al., 1998). A partir da ativação e da agregação de plaquetas, vários fatores de coagulação são ativados no local da lesão, gerando como produto final a fibrina (Hagberg et al., 1998). Dessa forma, durante a hemostasia, a atividade das plaquetas caracteriza-se por ativação, adesão, secreção e agregação (Klinger, 1997). A agregação pode ser reversível ou irreversível, dependendo do estímulo ou da concentração de agente agonista (Meyer et al., 1982).

As plaquetas podem ligar-se a polimorfonucleares, monócitos e alguns linfócitos que apresentam a proteína de ligação à P-selectina na presença de fibrinogênio (Weiss et al., 1998). Essa interação é reversível e independente da ativação do outro tipo celular, embora possa desencadeá-la (Hagberg et al., 1998). A ligação entre plaquetas e neutrófilos tem por objetivo estabelecer algumas atividades de cooperação, como a síntese transcelular de lipoxinas e a produção de imunoglobulinas (Klinger, 1997). O cininogênio de alto peso molecular (HMWK) pode ser liberado dos grânulos das plaquetas ativadas e é capaz de ativar a via intrínseca da coagulação e, conseqüentemente, a via do complemento, assim como o sistema das cininas, que, por sua vez, ativam o fator de Hageman, criando um ciclo de amplificação das reações (Cotran et al., 2000).

Outro fator importante para a ativação de plaquetas é o contato com toxinas como leuco (Nyarko et al., 1998) e endotoxinas (Jarvis e Evans, 1994) presentes na circulação sangüínea. Mesmo não havendo lesão endotelial, elas podem se aderir e promover agregação sobre o endotélio íntegro (Rosenblum, 1997; Reininger et al., 1998).
A função da ativação das plaquetas e da agregação intercelular na obstrução vascular ou trombose é desconhecida, entretanto agregados de plaquetas e neutrófilos foram encontrados em eqüinos com laminite na fase prodrômica (Weiss et al., 1997). Embora a laminite tenha sido definida como a inflamação das lâminas sensitivas do casco, hoje se acredita que ela seja uma doença vascular periférica, geralmente em conseqüência de outra doença sistêmica (IngleFehr e Baxter, 1998).

A fisiopatogenia da laminite inclui endotoxemia, vasoconstrição nas lâminas dérmicas, formação de anastomoses (Robinson, 1990) e trombose capilar (Weiss et al., 1997), o que ocasiona alteração das "forças de Starling" e, conseqüentemente, edema (Allen et al., 1990; Eaton et al., 1995). Segundo Byars et al. (2003), a hipercoagulação, que inclui trombocitose e trombose, pode estar envolvida na fisiopatogenia da laminite e deve ser avaliada juntamente com os testes de coagulação. Weiss et al. (1995) indicaram que o início do consumo de plaquetas ocorre seis horas após a indução da laminite com carboidratos. Dessa forma, o consumo e a deposição de plaquetas poderiam resultar de sua ativação primária na circulação sangüínea.

Os antiinflamatórios não-esteroidais podem inibir a agregação de plaquetas por acetilar a enzima ciclo-oxigenase de forma reversível (Heath et al., 1998) ou irreversível (Heath et al., 1994; Moses e Bertone, 2002), entretanto a função dessas drogas na ativação de plaquetas permanece pouco conhecida. Bastos da Silva et al. (1998) avaliaram o efeito do WEB 2086 antagonista do $P A F$ - e do ketoprofeno em inibir a agregação plaquetária, mas não estudaram a morfologia das plaquetas, do mesmo modo que Meyers et al. (1979), que avaliaram os efeitos da fenilbutazona.

Segundo Villamandos et al. (1996), poucos estudos têm sido desenvolvidos para avaliar a mudança de forma das plaquetas na patogenia de doenças virais e hemorrágicas.

O objetivo deste trabalho foi avaliar a morfologia das plaquetas no curso de desenvolvimento da laminite aguda induzida por sobrecarga de carboidratos e após o tratamento com fenilbutazona, flunixin meglumina e ketoprofeno. 


\section{MATERIAL E MÉTODOS}

Foram utilizados 20 eqüinos hígidos, sem raça definida, machos castrados, com idade entre três e 15 anos, peso entre 264 e $344 \mathrm{~kg}$ e escore corporal de dois a três. Previamente ao experimento, os animais passaram por um período de quarentena, quando foram avaliados, selecionados e, posteriormente, pesados e numerados. Eles permaneceram em piquete até sete dias antes da indução da laminite, quando foram instalados em baias individuais, com cama de serragem, onde receberam água à vontade, feno $^{1}$ e ração concentrada ${ }^{2}$, duas vezes ao dia, em quantidades equivalentes a $2,5 \%$ e $1 \%$ de peso corporal, respectivamente. À ração foram adicionados $50 \mathrm{~g}$ de sal mineral para eqüinos ${ }^{3}$.

Sete dias antes da indução experimental, foram privados da ração comercial e passaram por controle de endo e ectoparasitos, administrandose uma base conjugada de ivermectina e pomoatos de praziquantel e pirantel $^{4} \mathrm{e}$ deltametrina ${ }^{5}$.

Após a indução da laminite, os animais receberam suplemento eletrolítico ${ }^{6}(25 \mathrm{~g} / 10 l$ de água) adicionado diariamente à água de beber.

A indução de laminite foi feita pela administração de carboidratos, $20 \mathrm{~g}$ de amido de milho $/ \mathrm{kg}$ de peso corporal, diluídos em água, segundo modificação do modelo experimental utilizado por Weiss et al. (1998). Trinta e seis horas após a indução da laminite, os eqüinos, divididos aleatoriamente em quatro grupos experimentais, receberam a cada 12 horas: solução salina (GC) $0,9 \%^{8} 10 \mathrm{ml}$, ketoprofeno ${ }^{9}$ (GK) $2,2 \mathrm{mg} / \mathrm{kg}$, fenilbutazona ${ }^{10}$ (GF) $4,4 \mathrm{mg} / \mathrm{kg}$ ou flunixin meglumina ${ }^{11}$ (GFM) $1,1 \mathrm{mg} / \mathrm{kg}$.

As amostras de sangue foram colhidas antes e a cada 12 horas, até 72 horas após a indução da laminite, em horários fixos. Realizou-se uma

\footnotetext{
Coast Cross (Cynodon dactylon).

${ }^{2}$ Nutriage-Guabi (15\% de proteína), Brasil

${ }^{3}$ Centauro 80- Guabi, Brasil.

${ }^{4}$ Centurion-Valleé, Brasil.

${ }^{5}$ Butox-Intervet AS, Brasil.

${ }^{6}$ Plusvital.Marcolab, Brasil.

${ }^{7}$ Milhena- Nutril Brasil.

${ }^{8}$ Solução salina 0,9\%- Sanobiol. Brasil.

${ }^{9}$ Ketofen- Merial. Brasil.

${ }^{10}$ Equipalazone- Marcolab. Brasil.

${ }^{11}$ Flumegan- Marcolab. Brasil.
}

avaliação extra com seis horas, para melhor acompanhar a fase prodrômica da laminite, somando, portanto, oito tempos de avaliação ( $0 \mathrm{~h}$, 6h, 12h, 24h, 36h, 48h, 60h e 72h). Foram obtidas por venopunção jugular utilizando-se de frascos a vácuo contendo citrato de $\operatorname{sódio}^{12}$ a $3,8 \%$ (uma parte de citrato para nove de sangue). Após desprezar os três primeiros mililitros de sangue, as amostras foram centrifugadas ${ }^{13}$ no laboratório de análises clínicas a 1000rpm (260g) por cinco minutos. O sobrenadante, separado e adicionado de $1 \%$ de fixador, foi centrifugado por mais 20 minutos a 2000rpm (520g). Após o descarte do sobrenadante, fixou-se o sedimento com fixador Karnovsky ${ }^{14}$ e com tetróxido de ósmio a 0,1M. Após três lavagens sucessivas com tampão cacodilato $0,05 \mathrm{M}$, a amostra, précontrastada com acetato de uranila $0,5 \%$ durante 12 horas, foi desidratada em seqüência de diluições de acetona $(30,50,70,90,100,100$, $100 \%$ - 10 minutos cada) e polimerizada em metacrilato ${ }^{15}$ a $70^{\circ} \mathrm{C}$, por 48 horas.

As amostras polimerizadas foram cortadas em ultramicrótomo $^{16}$, acondicionadas em telas de níquel ${ }^{17}$ de 75 mesh e contrastadas com acetato de uranila $3 \%$ e com citrato de chumbo a $10 \%$, cada um por 10 minutos.

As alterações morfológicas das plaquetas foram classificadas, segundo Wurzinger e SchmidSchönbein (1990), em: 0- normal- ligeiramente oval, com organelas uniformemente dispersas no citoplasma; 1- incerta- perfil arredondado, organelas não centralizadas; 2- ativada- forma oval irregular com centralização de organelas e emissão de pseudópodes; 3- com alteração irreversível- discóide com organelas na periferia; 4- com lise total- perda da arquitetura normal.

A classificação das plaquetas foi em porcentagem, a partir da contagem total de 300 células de cada amostra, em microscópio eletrônico de transmissão ${ }^{18}$. Foram consideradas macroplaquetas quando o perímetro excedia $\mathrm{o}$

\footnotetext{
${ }^{12}$ Vacuette. Greiner Bio-one. Brasil.

${ }^{13}$ Labofuge 400- Heraeus- D- 6450. Alemanha.

14 Karnovsky (2,5\% gluteraldeído, 2,5\% paraformaldeído diluídos em tampão fosfato $0,05 \mathrm{M}$ com $\mathrm{pH}$ ajustado em 7,0 com a adição de cloreto de cálcio $0,001 \mathrm{M}$ )

${ }^{15}$ Spurr. Sigma. Aldrich química. Brasil.

${ }^{16}$ Reicher. Ultracut E. Leica. Alemanha

${ }^{17}$ Agar Scientific. Alemanha

${ }^{8}$ Zeiss EM 900. Alemanha.
} 
dobro da média do perímetro das plaquetas consideradas ativadas.

Imagens digitalizadas de 20 plaquetas selecionadas das amostras acima mencionadas foram processadas pelo programa de editoração de imagens $\mathrm{KS}_{300}{ }^{19}$ versão 2.0. Mensuraram-se a área, o perímetro e os eixos maior e menor das plaquetas, o número de organelas e o espaço por elas ocupado. Aferiu-se, também, o espaço ocupado pelos grânulos de glicogênio encontrados.

As variáveis analisadas foram comparadas de forma não pareada pelo teste $\mathrm{t}$ de Student, utilizando-se o programa estatístico GraphPad/Prism 3.0, com grau de significância de $5 \%$.

\section{RESULTADOS E DISCUSSÃO}

As médias das plaquetas normais e ativadas encontram-se nas Tab. 1 e 2. Em estado normal, as plaquetas de eqüinos apresentaram área de $5,33 \pm 1,34 \mu \mathrm{m}^{2}$, perímetro de $7,67 \pm 1,37 \mu \mathrm{m}$ e eixos maior e menor de $2,18 \pm 1,16 \mu \mathrm{m}$ e $1,76 \pm 1,45 \mu \mathrm{m}$, respectivamente (Fig. 1).

A área das plaquetas foi maior que a descrita por Wurzinger (1990) em plaquetas do homem e semelhante à encontrada por Meyers et al. (1982) em bovinos. Segundo Bastos da Silva et al. (1998), o sistema de canalículos abertos (SCA) está ausente em plaquetas de bovinos, o que pode representar a necessidade de mecanismos especiais de absorção e secreção de substâncias por essas células. O SCA não foi encontrado nas plaquetas deste estudo, o que pode sugerir deficiência ou ausência deles nas plaquetas de eqüinos, de forma semelhante à de bovinos. Este resultado poderia ainda explicar o fato de as plaquetas de eqüinos serem menos sensíveis à indução da agregação, com baixas concentrações de ADP, necessitando de concentrações muitas vezes superiores à utilizada para humanos.

Apesar de Weiss et al. (1997) considerarem a agregação heterotípica de plaquetas importante na patogenia da laminite, este achado foi observado em apenas $15 \%$ das amostras, sendo considerado esporádico (Fig. 2). Neste

\footnotetext{
${ }^{19}$ Kontrol Elektronic GMBH. Alemanha.
}

experimento, usaram-se amostras in vivo, enquanto Weiss et al. (1997) avaliaram a propensão de amostras in vitro. Como a dinâmica do fluxo sangüíneo pode influenciar a interação celular e as concentrações de moléculas e substâncias (Meyers et al., 1979; Kaneko et al., 1997; Nyarko et al., 1998), a avaliação das interações pode ser prejudicada pela falta da dinâmica do fluxo sangüíneo in vitro.

Embora Rinder et al. (1991a) tenham considerado a adesão entre plaquetas e linfócitos mais rara do que com outros leucócitos, este achado foi observado em $50 \%$ das amostras em que a agregação heterotípica ocorreu (Fig. 3). Alguns autores acreditam que a laminite seja uma reação local do tipo Schwartzman, desencadeada pela endotoxina, e que as plaquetas, por serem extremamente sensíveis a qualquer alteração física ou química do sangue ou do sistema cardiovascular, responderiam prontamente ao estímulo (Wurzinger, 1990; Jarvis e Evans, 1994; Klinger, 1997).

Mitocôndrias foram observadas em apenas 10\% das plaquetas, número inferior ao citado por Wurzinger (1990), em humanos, e por Bastos da Silva et al. (1998), em suínos. Os grânulos de glicogênio (Fig. 4) foram um achado constante e superior em plaquetas ativadas que em plaquetas normais (Tab. 2). Este resultado pode ser atribuído ao consumo de energia para ativação, emissão de pseudópodes e interação celular. Bastos da Silva et al. (1998) descreveram diferenças entre plaquetas normais e ativadas de bovinos durante a ativação pelo fator de agregação plaquetário (PAF) e mencionaram que as plaquetas ativadas apresentam alterações no sistema de microtúbulos, degranulação dos $\gamma$ grânulos, aumento no número de $\alpha$-grânulos e de mitocôndrias e ausência de grânulos de glicogênio.

Neste estudo, houve estímulo in vivo à ativação das plaquetas por fatores relacionados à fisiopatogenia da laminite que, segundo Jarvis e Evans (1994), estão principalmente ligados à endotoxemia. Segundo Weiss et al. (1997), a endotoxina ativa mecanismos relacionados ao PAF, estimula e desencadeia a reação de Schwartzman, já mencionada (Allen et al., 1990; Eaton et al., 1995) e relacionada à hipercoagulação (Byars et al., 2003). 
$\mathrm{O}$ resultado mais comum em plaquetas ativadas de eqüinos foi a emissão de pseudópodes (Fig. 5 e 6) que, segundo Klinger (1997), aumenta a área de contato intercelular e é acompanhada da expressão e redistribuição de glicoproteínas da membrana.

Tabela 1. Médias e desvios-padrão da área, perímetro e eixos maior e menor de plaquetas normais e ativadas de eqüinos

\begin{tabular}{lcccc}
\hline Plaqueta & Área $\left(\mu \mathrm{m}^{2}\right)$ & Perímetro $(\mu \mathrm{m})$ & Eixo maior $(\mu \mathrm{m})$ & Eixo menor $(\mu \mathrm{m})$ \\
\hline Normal & $5,33 \pm 1,34 \mathrm{a}$ & $7,67 \pm 1,37 \mathrm{a}$ & $2,18 \pm 1,16 \mathrm{a}$ & $1,76 \pm 1,45 \mathrm{a}$ \\
Ativada & $6,02 \pm 1,64 \mathrm{a}$ & $10,65 \pm 1,49 \mathrm{~b}$ & $3,67 \pm 0,83 \mathrm{~b}$ & $2,27 \pm 0,29 \mathrm{a}$ \\
\hline
\end{tabular}

Letras diferentes na mesma coluna representam diferença $(\mathrm{P}<0,05)$ entre plaquetas normais e ativadas.

Tabela 2. Médias e desvios-padrão da área, perímetro e eixos maior e menor das organelas de plaquetas normais e ativadas de eqüinos

\begin{tabular}{lcccccc}
\hline Plaqueta & Organela & $\begin{array}{c}\text { Área } \\
\left(\mu \mathrm{m}^{2}\right)\end{array}$ & $\begin{array}{c}\text { Perímetro } \\
(\mu \mathrm{m})\end{array}$ & $\begin{array}{c}\text { Eixo maior } \\
(\mu \mathrm{m})\end{array}$ & $\begin{array}{c}\text { Eixo menor } \\
(\mu \mathrm{m})\end{array}$ & $\begin{array}{c}\mathrm{N}^{\circ} / \\
\text { plaqueta }\end{array}$ \\
\hline \multirow{4}{*}{ Normal } & mitocôndria & $0,12 \pm 0,1 \mathrm{a}$ & $0,22 \pm 0,02 \mathrm{a}$ & $0,04 \pm 0,12 \mathrm{a}$ & $0,01 \pm 0,001 \mathrm{a}$ & $0,2 \mathrm{a}$ \\
& $\alpha$-grânulo & $0,05 \pm 0,04 \mathrm{a}$ & $1,19 \pm 0,44 \mathrm{a}$ & $0,34 \pm 0,24 \mathrm{a}$ & $0,30 \pm 0,11 \mathrm{a}$ & $8 \mathrm{a}$ \\
& $\gamma$-grânulo & $0,28 \pm 0,2 \mathrm{a}$ & $2,93 \pm 0,1 \mathrm{a}$ & $0,78 \pm 0,01 \mathrm{a}$ & $0,57 \pm 0,001 \mathrm{a}$ & $0,2 \mathrm{a}$ \\
& glicogênio & $0,13 \pm 0,05 \mathrm{a}$ & $1,48 \pm 0,59 \mathrm{a}$ & - & - & - \\
\multirow{4}{*}{ Ativada } & mitocôndria & $0,28 \pm 0,02 \mathrm{a}$ & $0,43 \pm 0,1 \mathrm{a}$ & $0,01 \pm 0,001 \mathrm{a}$ & $0,01 \pm 0,001 \mathrm{a}$ & $0,2 \mathrm{a}$ \\
& $\alpha$-grânulo & $0,10 \pm 0,04 \mathrm{a}$ & $1,58 \pm 0,27 \mathrm{a}$ & $0,34 \pm 0,07 \mathrm{a}$ & $0,39 \pm 0,06 \mathrm{a}$ & $6 \mathrm{a}$ \\
& $\gamma$-grânulo & $0,06 \pm 0,12 \mathrm{~b}$ & $1,30 \pm 0,05 \mathrm{~b}$ & $0,32 \pm 0,0 \mathrm{a}$ & $0,27 \pm 0,01 \mathrm{~b}$ & $3 \mathrm{~b}$ \\
& glicogênio & $0,16 \pm 0,0,01 \mathrm{~b}$ & $1,78 \pm 1,24 \mathrm{a}$ & - & - & - \\
\hline
\end{tabular}

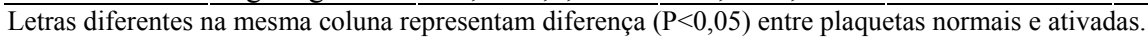

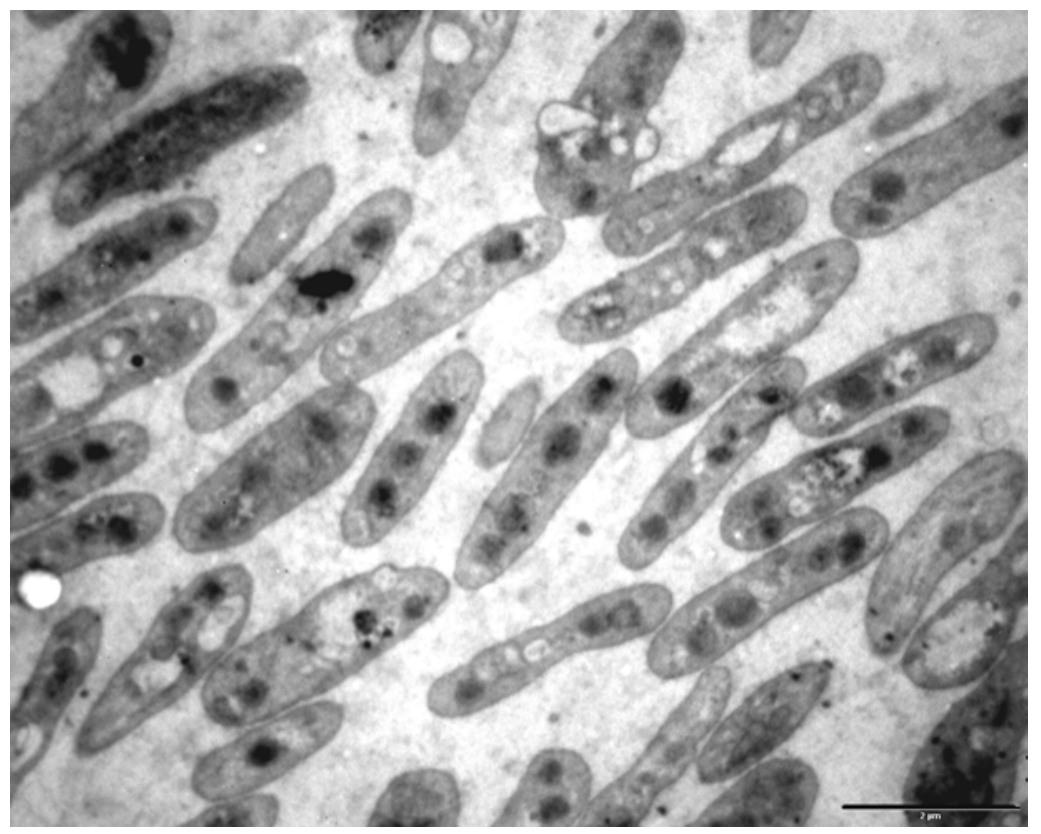

Figura 1. Fotografia ultra-estrutural, mostrando vista panorâmica de plaquetas normais, não ativadas. A barra representa $2 \mu \mathrm{m}$. 


\section{Paes Leme et al.}

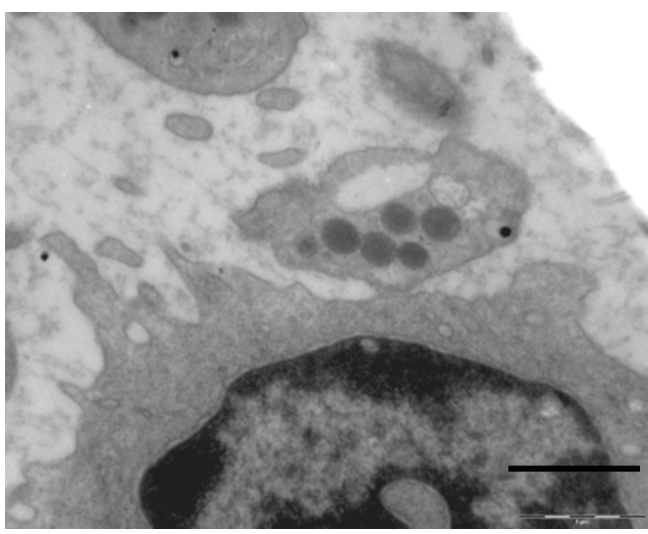

Figura 2. Fotografia ultra-estrutural, mostrando agregação heterotípica entre plaqueta e leucócito polimorfonuclear (PMN). A barra representa $1 \mu \mathrm{m}$.

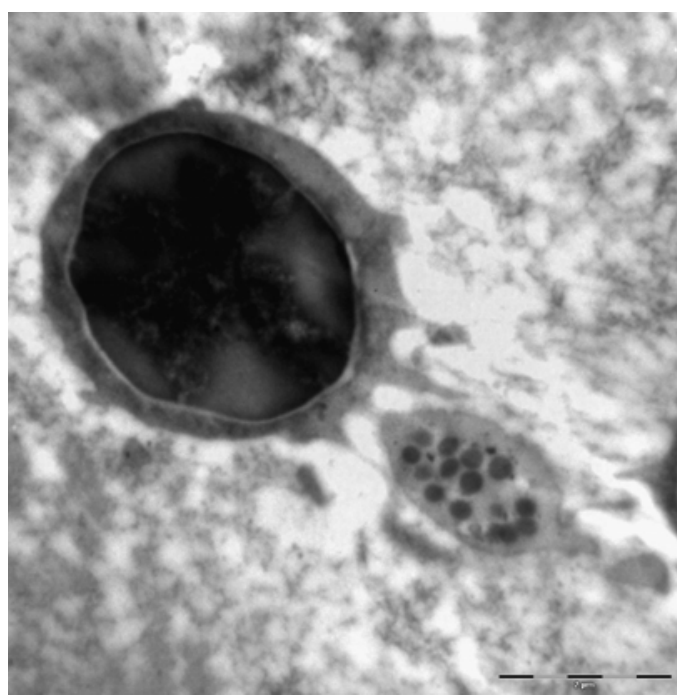

Figura 3. Fotografia ultra-estrutural, mostrando interação entre plaqueta e linfócito. A barra representa $2 \mu \mathrm{m}$.

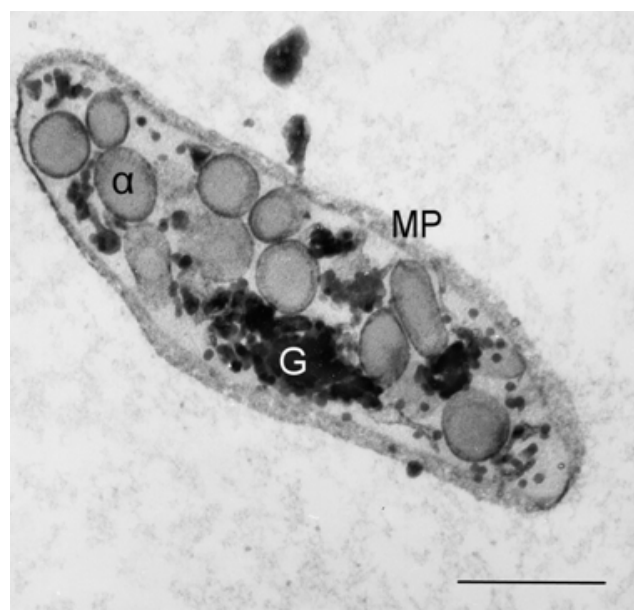

Figura 4. Fotografia ultra-estrutural de plaqueta normal de eqüino, mostrando presença de grânulos de glicogênio (G) em abundância. $\alpha$ - representa $\alpha$ grânulos e MP, membrana plasmática. A barra representa $1 \mu \mathrm{m}$.

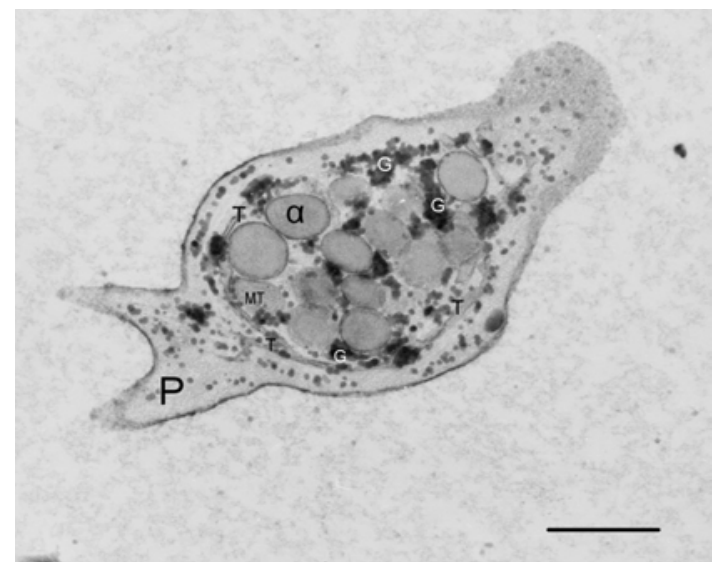

Figura 5. Fotografia ultra-estrutural de plaqueta de eqüino ativada, mostrando emissão de pseudópodes. $\alpha$ - grânulos, MT- mitocôndria, T- microtúbulos, Gglicogênio. A barra representa $1 \mu \mathrm{m}$.
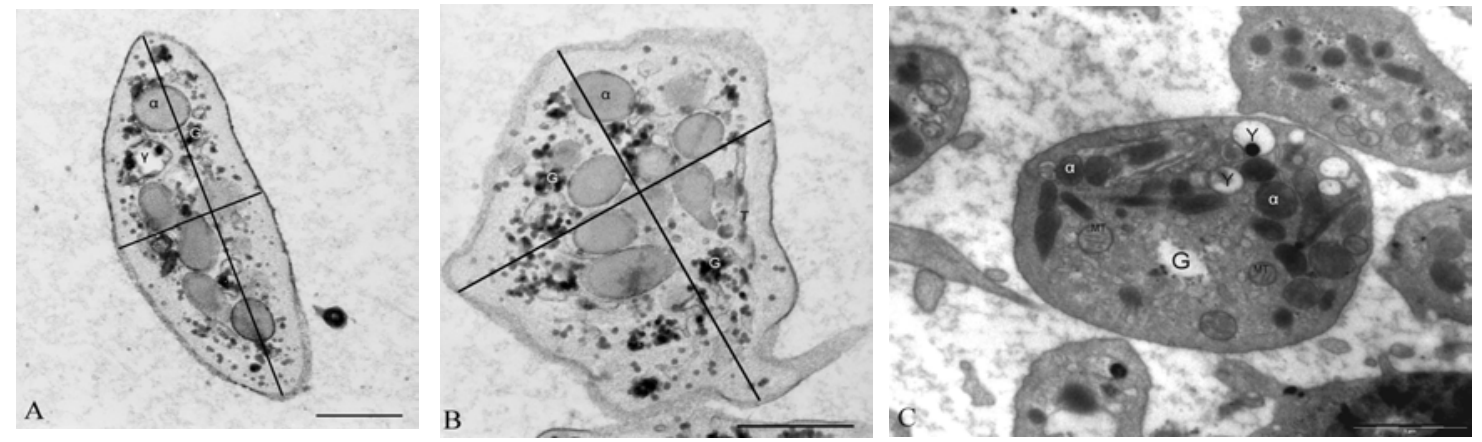

Figura 6. Fotografia ultra-estrutural, mostrando a medida dos eixos maior e menor de plaquetas normais (A), ativadas (B) e estado incerto $(\mathbf{C})$. Y = gama grânulos, $\alpha=$ alfa grânulos, MT= mitocôndria, $\mathrm{G}=$ grânulos de glicogênio ou área onde se encontrariam. Em A e B a barra representa 200nm. Em C a barra representa $1 \mu \mathrm{m}$. 
As alterações observadas nas plaquetas deste estudo incluíram, além da mudança de forma, alteração da relação entre os eixos maior e menor (Fig 6), aumento do perímetro, emissão de pseudópodes, aumento no número de $\alpha$-grânulos e redução no número de $\gamma$-grânulos e grânulos de glicogênio. Estes resultados eram esperados e foram semelhantes ao encontrado por Bastos da Silva et al. (1998). Um achado que não foi descrito por Bastos da Silva et al. (1998) foi a diminuição do tamanho dos $\alpha$ e $\gamma$-grânulos, que pode representar o aumento do consumo ou secreção dos constituintes por eles armazenados.

A presença de macroplaquetas ocorreu em $80 \%$ das amostras analisadas. Segundo Bastos da Silva et al. (1998), a presença de macroplaquetas pode caracterizar uma resposta medular a estímulos como perda de sangue ou consumo de plaquetas.

Não foram observadas lises celulares ou plaquetas consideradas em estádio irreversível de ativação, como o encontrado por Wurzinger e Schmid-Schönbein (1990). Esses autores verificaram lise de plaquetas após exposição a uma força de cisalhamento equivalente ou superior a $170 \mathrm{~N} / \mathrm{m}^{2}$. A rotação empregada neste experimento, para obtenção do PRP, não ocasionou tal achado. Dessa forma, pode-se dizer que a metodologia empregada neste estudo foi adequada para a obtenção de amostras de plaquetas de eqüinos e que a laminite parece não resultar em alterações irreversíveis de plaquetas.

A ativação plaquetária iniciou seis horas após a administração do carboidrato (Tab. 3; Fig. 7). Segundo Weiss et al. (1995), o consumo de plaquetas, seis horas após a indução da laminite com carboidratos, poderia resultar de sua ativação primária na circulação sangüínea. A ativação das plaquetas permaneceu inalterada entre 24 e 36 horas após a indução da laminite, entretanto os sinais clínicos da laminite puderam ser observados entre 24 e 36 horas. Isso sugere que as alterações plaquetárias são importantes na fase prodrômica da laminite e podem desencadeá-la ou potencializá-la.

Tabela 3. Estado morfológico das plaquetas (\%) em eqüinos com laminite induzida por sobrecarga de carboidratos

\begin{tabular}{lccc}
\hline Tempo & Normal & Incerta & Ativada \\
\hline $0 \mathrm{~h}$ & $62,4 \pm 10,5 \mathrm{Aa}$ & $18,0 \pm 3,1 \mathrm{Ab}$ & $19,6 \pm 8,7 \mathrm{Cb}$ \\
$6 \mathrm{~h}$ & $46,8 \pm 14,0 \mathrm{Aa}$ & $18,6 \pm 4,5 \mathrm{Ab}$ & $34,6 \pm 16 \mathrm{Bab}$ \\
$12 \mathrm{~h}$ & $29,0 \pm 3,8 \mathrm{BCb}$ & $13,6 \pm 7,1 \mathrm{Ac}$ & $57,4 \pm 6,9 \mathrm{Aa}$ \\
$24 \mathrm{~h}$ & $23,8 \pm 6,7 \mathrm{Cb}$ & $18,2 \pm 6,8 \mathrm{Ab}$ & $58,0 \pm 2,2 \mathrm{Aa}$ \\
$36 \mathrm{~h}$ & $30,0 \pm 12,4 \mathrm{Bb}$ & $10,6 \pm 3,7 \mathrm{Ac}$ & $59,4 \pm 16 \mathrm{Aa}$ \\
\hline
\end{tabular}

Letras maiúsculas na mesma coluna representam diferença $(\mathrm{P} \leq 0,05)$ entre tempos. Letras minúsculas na mesma linha representam diferença $(P \leq 0,05)$ entre estados morfológicos.

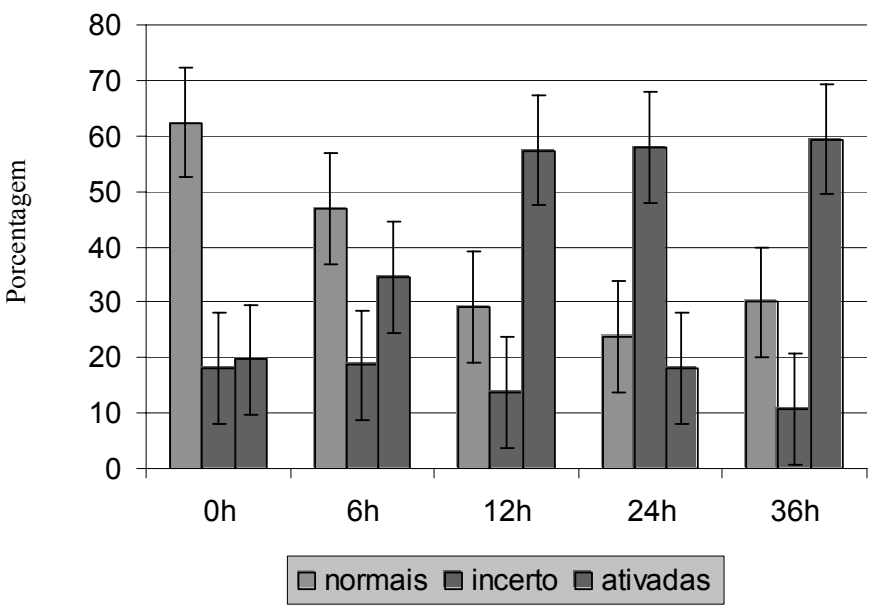

Figura 7. Estado morfológico das plaquetas (\%) em eqüinos com laminite induzida por sobrecarga de carboidratos. 
Após 12 horas de medicados (48 horas da administração do carboidrato), notou-se diferença estatística entre os animais dos grupos tratados e controle (Tab. 4; Fig. 8), sugerindo que as três drogas utilizadas inibiram a ativação de plaquetas de eqüinos com laminite induzida. Entretanto, com 24 horas de tratamento (60 horas da administração do carboidrato), notaram-se melhores resultados nas amostras dos animais dos GF e GFM. Com 36 horas de tratamento ( 72 horas da administração do carboidrato), apesar de não haver diferença estatística, GK e GFM apresentaram melhores resultados que GF. Em todos os tempos de tratamento, os resultados de $\mathrm{GC}$ foram inferiores aos dos grupos tratados.

Tabela 4. Estado morfológico das plaquetas (\%) em eqüinos com laminite induzida por sobrecarga de carboidratos e tratados com ketoprofeno (GK) fenilbutazona (GF), flunixin meglumina (GFM) ou solução salina (GC)

\begin{tabular}{|c|c|c|c|c|}
\hline \multirow{2}{*}{ Tempo } & \multirow{2}{*}{ Grupo } & \multicolumn{3}{|c|}{ Estado morfológico de plaquetas (\%) } \\
\hline & & Normal & Incerta & Ativada \\
\hline \multirow{4}{*}{$48 \mathrm{~h}$} & GK & $26,8 \pm 12,1 \mathrm{Ab}$ & $13,2 \pm 1,1 \mathrm{Ab}$ & $60 \pm 12,6 \mathrm{Aa}$ \\
\hline & GF & $23 \pm 9,7 \mathrm{Ab}$ & $11,8 \pm 4,7 \mathrm{Ab}$ & $65,2 \pm 14,3 \mathrm{Aa}$ \\
\hline & GFM & $25,6 \pm 6,6 \mathrm{Ab}$ & $15,6 \pm 3,2 \mathrm{Ac}$ & $58,8 \pm 6,7 \mathrm{Aa}$ \\
\hline & $\mathrm{GC}$ & $19,2 \pm 2,6 \mathrm{Bb}$ & $14,4 \pm 2,5 \mathrm{Ab}$ & $66,4 \pm 4,6 \mathrm{Aa}$ \\
\hline \multirow{4}{*}{$60 \mathrm{~h}$} & GK & $21,6 \pm 5,3 \mathrm{Ab}$ & $12,2 \pm 3,3 \mathrm{Ac}$ & $66,2 \pm 3,9 \mathrm{Aa}$ \\
\hline & GF & $37 \pm 8,9 \mathrm{Aa}$ & $11,8 \pm 5,6 \mathrm{Ab}$ & $51,2 \pm 9,9 \mathrm{Aa}$ \\
\hline & GFM & $33 \pm 13,7 \mathrm{Aa}$ & $14,6 \pm 4,3 \mathrm{Ab}$ & $52,4 \pm 16,5 \mathrm{Aa}$ \\
\hline & GC & $24,6 \pm 12,2 \mathrm{ABb}$ & $13,2 \pm 5,8 \mathrm{Ab}$ & $62,2 \pm 17,6 \mathrm{Aa}$ \\
\hline \multirow{4}{*}{$72 \mathrm{~h}$} & GK & $33,4 \pm 6,5 \mathrm{Ab}$ & $15,8 \pm 5,4 \mathrm{Ac}$ & $50,8 \pm 10,8 \mathrm{Aa}$ \\
\hline & GF & $25 \pm 14,3 \mathrm{Ab}$ & $14 \pm 2,0 \mathrm{Ac}$ & $61 \pm 14,8 \mathrm{Aa}$ \\
\hline & GFM & $29,4 \pm 16,1 \mathrm{Aab}$ & $13,8 \pm 4,2 \mathrm{Ab}$ & $56,8 \pm 18,7 \mathrm{Aa}$ \\
\hline & GC & $28,6 \pm 13,7 \mathrm{Ab}$ & $9,2 \pm 5,5 \mathrm{Ac}$ & $62,2 \pm 17,7 \mathrm{Aa}$ \\
\hline
\end{tabular}

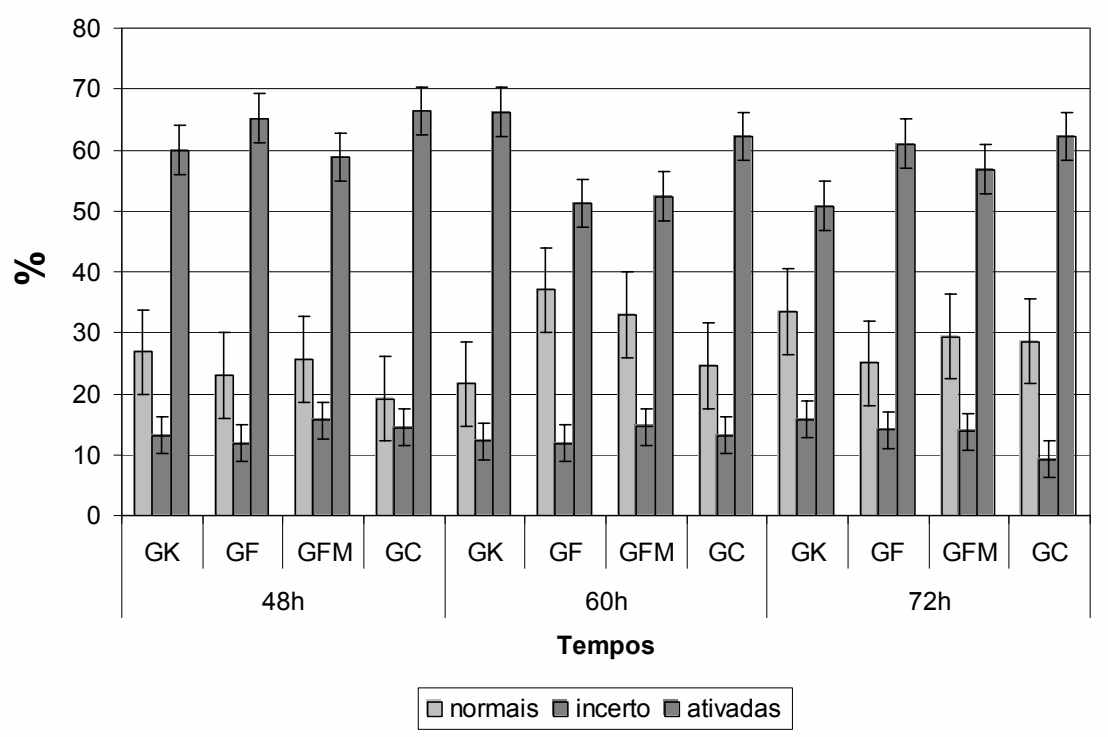

Figura 8. Estado morfológico das plaquetas (\%) em eqüinos com laminite induzida por sobrecarga de carboidratos e tratados com ketoprofeno (GK) fenilbutazona (GF), flunixin meglumina (GFM) ou solução salina (GC).

Segundo Heath et al. (1994), Heath et al. (1998) e Moses e Bertone (2002), os antiinflamatórios não-esteroidais podem inibir a agregação de plaquetas ao acetilar as enzimas ciclooxigenases. Neste trabalho, os antiinflamatórios não-esteroidais reduziram a ativação das plaquetas de eqüinos in vivo, sendo o flunixin meglumina superior quando se compararam plaquetas normais e ativadas (Tab. 4). Houve equivalência entre plaquetas normais e ativadas por período mais prolongado (72 horas) nas amostras obtidas dos animais do GFM em relação aos do GK e GF. Talvez este resultado esteja relacionado à atividade antiendotoxêmica do flunixin meglumina (Moses e Bertone, 2002) que, por reduzir a atividade da endotoxina, incorre em menores taxas de ativação plaquetária. 
A ativação e a agregação plaquetárias in vitro podem diferir dos achados in vivo, já que se faz necessário um controle da contagem celular nas amostras, reduzindo a interação celular. Neste experimento, pôde-se notar que as plaquetas, quando consideradas ativadas, apresentam perfil de organela diferente do perfil de plaquetas normais. Os fármacos antiinflamatórios nãoesteroidais inibem a ativação plaquetária de eqüinos in vivo, sendo a atividade do flunixin meglumina mais prolongada do que a da ketoprofeno e fenilbutazona.

\section{REFERÊNCIAS BIBLIOGRÁFICAS}

ABLETT, J.M.; FAIRBAIRN, S.M.; PAGE, C.P. et al. Influence of antigen challenge on platelet responsiveness in horses with chronic obstructive pulmonary disease. Equine Vet. J., v.29, p.382-6, 1997.

ALLEN, D.; CLARK, E.S.; MOORE, J.N. et al. Evaluation of equine digital Starling forces and hemodynamics during early laminitis. Am. J. Vet. Res., v.51, p.1930-1934, 1990.

BASTOS DA SILVA, M.; DESSY, C.; COGHE, J. et al. Protective effects of WEB 2086 (PAF antagonist) and ketoprofen (NSAID) on PAF induced changes in the morphological ultra structure of blood platelets in calves. Vet. Res. Comm., v.22, p.273-291, 1998.

BYARS, T.D.; DAVIS, D.; DIVERS, T.J. Coagulation in the equine intensive care patient. Clin. Technol. Equine Pract., v.2, p.178-187, 2003.

COTRAN, R.S.; KUMAR, V.; COLLINS, T. Robbins patologia estrutural e funcional. 4.ed. Rio de Janeiro: Guanabara Koogan, 2000. 1251p.

EATON, S.A.; ALLEN, D.; EADES, S.C. et al. Digital Starling forces and hemodynamics during early laminitis induced by an aqueous extract of black walnut (Juglans nigra) in horses. Am. J. Vet. Res., v.56, p.1338-1344, 1995.

HAGBERG, I.A.; ROALD, H.E.; LIZBERG, T. Adhesion of leukocytes to growing arterial thrombi. Thromb. Haemostasis, v.80, p.852-858, 1998.

HEATH, M.F.; EVANS, R.J.; HAYES, L.J. Dextran 70 inhibits equine platelet aggregation induced by PAF but not by others agonists. Equine Vet. J., v.30, p.408-411, 1998.

HEATH, M.F.; EVANS, R.J.; POOLE, A.W. et al. The effects of aspirin and paracetamol on the aggregation of equine blood platelets. J. Vet. Pharm. Therap., v.17, p.374$378,1994$.

INGLE FEHR, J.E.; BAXTER, G.M. Evaluation of digital and laminar blood flow in horses given a low dose of endotoxin. Am. J. Vet. Res., v.59, p.192-196, 1998.

JARVIS, G.E.; EVANS, R.J. Endotoxin induced platelet aggregation in heparinised equine whole blood in vitro. Res. Vet. Sci., v.57, p.317-24, 1994.
KANEKO, J.J.; HARVEY, J.W.; BRUSS, M.L. Clinical biochemistry of domestic animals. 5.ed. San Diego: Academic, 1997. 932p.

KLINGER, M.H.F. Platelets and inflammation. Anat. Embryol., v.196, p.1-11, 1997.

MEYERS, K.M.; HOLSEN, H.; SEACHORD, C.L. Comparative study of platelet dense granule constituents. Am. J. Physiol., v.243, p.454-461, 1982.

MEYERS, K.M.; LINDNER, C.; GRANT, B. Characterization of the equine platelet aggregation response. Am. J. Vet. Res., v.40, p.260-264, 1979.

MOSES, V.S.; BERTONE, A.L. Nonsteroidal antiinflammatory drugs. Vet. Clin. Equine, v.18, p.21-37, 2002.

NYARKO, K.A.; COOMBERS, B.L.; MELLORS, A. et al. Bovine platelet adhesion is enhanced by leukotoxin and sialoglycoprotease isolated from Pasteurella haemolytica A 1 cultures. Vet. Microbiol., v. 61, p. 81-91, 1998.

REININGER, A.; KORNDÖRFER, M.A.; WURZINGER, L.J. Adhesion of ADP- activated platelets to intact endothelium under stagnation point flow in vitro is mediated by integrin $\alpha \mathrm{IIb} \beta 3$. Thromb. Haemostasis, v.70, p.998-1003, 1998.

RINDER, H.M.; BONAN, J.L.; RINDER, C.S. et al. Activated und unactivated platelet adhesion to monocytes and neutrophils. Blood, v.78, p.1760-1769, 1991a.

RINDER, H.M.; BONAN, J.L.; RINDER, C.S. et al. Dynamics of leukocyte-platelet adhesion in whole blood. Blood, v.78, p.1730-1737, 1991b.

ROBINSON, N.E. Digital blood flow, arteriovenous anastomoses and laminitis. Equine Vet. J., v.22, p.381-383, 1990 .

ROSENBLUM, W.I. Platelet adhesion and aggregation without endothelial denudation or exposure of basal lamina and/or collagen. J. Vasc. Res., v.34, p.409-417, 1997.

VILLAMANDOS, J.C.; BAUTISTA, M.J.; HERVAS, J. et al. Subcellular changes in platelets in acute and subacute African swine fever. J. Comp. Pathol., v.118, p.327-341, 1996.

WEISS, D.J.; EVANSON, O.A.; MCCLENAHAN, D. et al., Effect of a competitive inhibitor of platelet aggregation on experimentally induced laminitis in ponies. Am. J. Vet. Res., v.59, p.814-817, 1998.

WEISS, D.J.; EVANSON, O.A.; WELLS, R.E. Evaluation of arginine-glycine-aspartate containing peptides as inhibitors of equine platelet function. Am. J. Vet. Res., v.5, p.457-460, 1997.

WEISS, D.J.; TRENT, A.M.; JOHNSTON, G. Prothrombotic events in the prodromal stages of acute laminitis in horses. Am. J. Vet. Res., v.56, p.986-991, 1995.

WURZINGER, L.J. Histophysiology of the circulating platelet. Adv. Anat. Embryo Cell Biol., v.120, p.1-96, 1990.

WURZINGER, L.J.; SCHMID-SCHÖNBEIN, H. The role of fluid dynamics in triggering and amplifying haemostatic reactions in thrombogenesis. Blood Flow Large Art., v.15, p.215-226, 1990. 